\title{
A COMMUTATIVE SEMISIMPLE ANNIHILATOR BANACH ALGEBRA WHICH IS NOT DUAL
}

BY B. E. JOHNSON

\author{
Communicated by E. Hewitt, December 7, 1966
}

Barnes [1] has constructed an example of a commutative semisimple normed annihilator algebra which is not a dual algebra. His example is not complete and when completed acquires a nonzero radical. In this paper we construct an example which is complete. The theory of annihilator algebras is developed e.g. in [2].

We put $\alpha_{i}=\left(1+(1+i)^{1 / 2}\right)^{-2}$ for $i \geqq 1$ and denote by $A_{0}$ the algebra of doubly infinite sequences $a$ with $a_{i}=0$ for all but a finite number of values of $i$, with coordinatewise addition and multiplication. We define a norm on $A_{0}$ by

$$
\|a\|=3\left(\sum_{n \leqq 0}\left|a_{n}\right|^{2}\right)^{1 / 2}+3 \sup _{n>0}\left|a_{n} \alpha_{n}^{-1}-\sum_{j=-n}^{0} a_{j}\right| .
$$

This is easily seen to be a linear space norm on $A_{0}$ and we have that

$$
\begin{aligned}
\left(\sum_{n \leqq 0}\left|a_{n} b_{n}\right|^{2}\right)^{1 / 2} & \leqq\left(\sum_{n \leqq 0}\left|a_{n}\right|^{2}\right)^{1 / 2}\left(\sum_{n \leqq 0}\left|b_{n}\right|^{2}\right)^{1 / 2} \\
& \leqq \frac{1}{9}\|a\|\|b\| ;
\end{aligned}
$$

(ii) if $n>0$,

$$
\begin{aligned}
\frac{1}{3}\|a\| & \geqq\left|a_{n} \alpha_{n}^{-1}-\sum_{j=-n}^{0} a_{j}\right| \\
& \geqq\left|a_{n}\right| \alpha_{n}^{-1}-(n+1)^{1 / 2} \frac{1}{3}|| a||
\end{aligned}
$$

so that

$$
\left|a_{n}\right| \alpha_{n}^{-1} \leqq \frac{1}{3}\left(1+(n+1)^{1 / 2}\right)\|a\|
$$

and

$$
\begin{aligned}
&\left|a_{n} b_{n} \alpha_{n}^{-1}\right| \leqq \frac{1}{9} \alpha_{n}\left(1+(n+1)^{1 / 2}\right)^{2}\|a\|\|b\| \\
&=\frac{1}{9}\|a\|\|b\| ; \\
&\left|\sum_{j=-n}^{0} a_{j} b_{j}\right| \leqq \\
&\left(\sum_{j=-n}^{0}\left|a_{j}\right|^{2}\right)^{1 / 2}\left(\sum_{j=-n}^{0}\left|b_{j}\right|^{2}\right)^{1 / 2} \\
& \leqq \frac{1}{9}\|a\|\|b\| .
\end{aligned}
$$


The submultiplicative property of $\|\cdot\|$ follows easily from (i), (ii) and (iii).

Consider now the space $l_{2}(-\infty, 0) \oplus c(1, \infty)$, which we consider as a space of doubly infinite sequences, with norm as the sum of the $l_{2}$ norm and the sup norm. For $a \in A_{0}$ define $T_{0} a$ by

$$
\begin{aligned}
\left(T_{0} a\right)_{n} & =3^{1 / 2} a_{n} \quad \text { for } n \leqq 0, \\
& =3 a_{n} \alpha_{n}^{-1}-3 \sum_{j=-n}^{0} a_{j} \quad \text { for } n>0 .
\end{aligned}
$$

$T_{0}$ is then a linear isometry; $A_{0} \rightarrow l_{2}(-\infty, 0) \oplus c(1, \infty)$. The multiplicative linear functionals on $A_{0}$ are

$$
\phi_{i}(a)=a_{i}
$$

and if the functionals $\psi_{i}$ on $l_{2} \oplus c$ are defined by

$$
\begin{aligned}
\psi_{i}(c) & =c_{i} / 3^{1 / 2} \quad \text { for } i \leqq 0, \\
& =\left(c_{i}+\left(3^{1 / 2}\right) \sum_{j=-i}^{0} c_{j}\right) \alpha_{i} / 3 \quad \text { for } i>0,
\end{aligned}
$$

then the $\psi_{i}$ are continuous and $T_{0}^{*} \psi_{i}=\phi_{i}$. The set $\left\{\psi_{i} ; i \in Z\right\}$ is clearly total on $l_{2} \oplus c$.

Let now $A$ be the completion of $A_{0} ; T_{0}$ extends to an isometry $T ; A \rightarrow l_{2} \oplus c$, the $\phi_{i}$ extend to multiplicative linear functionals on $A$ and $\phi_{i}=T^{*} \psi_{i}$. Since the $\psi_{i}$ are total on $l_{2} \oplus c$, the $\phi_{i}$ are total on $A$ and $A$ is a semisimple Banach algebra. Writing $a_{i}=\phi_{i}(a)$ for $a \in A$ we can consider the elements of $A$ as doubly infinite sequences and the two ways in which an element of $A_{0}$ becomes a sequence give the same sequence.

If $\delta_{i}$ is the sequence in $A_{0}$ with $\left(\delta_{i}\right)_{j}=\delta_{i j}$ (the Kronecker symbol) then $a \delta_{i}=a_{i} \delta_{i}$ for all $a$ in $A$ so that if $J$ is an ideal in $A$ either $\delta_{i} \in J$ or $\delta_{i} J=\{0\}$. Thus if $J$ is a closed ideal in $A$ with zero annihilator then all the $\delta_{i}$ are in $J, A_{0} \subset J$ and $J=A$. Hence $A$ is an annihilator algebra.

The span $J_{0}$ of the set $\left\{\delta_{i} ; i>0\right\}$ is an ideal in $A$ and thus so is its closure $J$. The annihilator of $J$ is

$$
K=\left\{b: b \in A, b_{i}=0 \text { for } i>0\right\}
$$

and the annihilator of $K$ is

$$
\tilde{J}=\left\{c: c \in A, c_{i}=0 \text { for } i \leqq 0\right\} .
$$

The norm on $\tilde{J}$ is given by $\|c\|=\sup \left|c_{i} / \alpha_{i}\right|$ and since $a_{n}=o\left(\alpha_{n}\right)$ as $n \rightarrow \infty$ for $a \in J_{0}$ we have $a_{n}=o\left(\alpha_{n}\right)$ for $a \in J$. Define a sequence $x_{n}$ from $A_{0}$ by 


$$
\begin{aligned}
\left(x_{n}\right)_{i} & =-1 /(n+1), \quad-n \leqq i \leqq 0, \\
& =0, \quad i<-n, \\
& =\left(1+\sum_{j=-i}^{0} x_{n j}\right) \alpha_{i}, \quad i>0 .
\end{aligned}
$$

Then, since the supremum term is 0 ,

$$
\begin{aligned}
\left\|x_{m}-x_{n}\right\| & =3\left(\sum_{i \leqq 0}\left|x_{m i}-x_{n i}\right|^{2}\right)^{1 / 2} \\
& \leqq 3\left(\sum_{i \leqq 0}\left|x_{m i}\right|^{2}\right)^{1 / 2}+3\left(\sum_{i \leqq 0}\left|x_{n i}\right|^{2}\right)^{1 / 2} \\
& =3(m+1)^{-1 / 2}+3(n+1)^{-1 / 2}
\end{aligned}
$$

so that $x_{n}$ converges to a limit $y$ in $A$. We have

$$
\begin{aligned}
y_{i}=\lim _{n} x_{n i} & =0, & & \text { if } i \leqq 0, \\
& =\alpha_{i}, & & \text { if } i>0 .
\end{aligned}
$$

Clearly $y \in \tilde{J}$ but $y_{i}=\alpha_{i} \neq 0\left(\alpha_{i}\right)$ so that $y_{i} \notin J$, the ideal $J$ is not an annihilator and $A$ is not a dual algebra.

The question of the existence of simple annihilator Banach algebras which are not dual remains open!

\section{REFERENCES}

1. B. A. Barnes, An annihilator algebra which is not dual, Bull, Amer. Math. Soc. 71 (1965), 573-576.

2. C. E. Rickart, General theory of Banach algebras, Van Nostrand, New York, 1960.

The University, Newcastle upon Tyne, England. 